
\title{
28 Research Square \\ Identification of the Terpenoid Compounds and Behavioral Assays of Alarm Pheromones in the Vetch Aphid Megoura Viciae
}

\section{Xuan Song}

China Agricultural University

Yao-Guo Qin

China Agricultural University

Yue Yin

China Agricultural University

Zheng-Xi Li ( $\square$ zxli@cau.edu.cn )

China Agricultural University https://orcid.org/0000-0003-3611-8046

\section{Research Article}

Keywords: Alarm pheromone, terpenoid compounds, behavioral assay, mixture formula, Megoura viciae

Posted Date: April 30th, 2021

DOI: https://doi.org/10.21203/rs.3.rs-467323/v1

License: () (i) This work is licensed under a Creative Commons Attribution 4.0 International License. Read Full License

Version of Record: A version of this preprint was published at Journal of Chemical Ecology on August 4th, 2021. See the published version at https://doi.org/10.1007/s10886-021-01297-4. 


\section{Abstract}

Aphids are destructive insect pests worldwide, and alarm pheromones play a key role in their chemical ecology. However, the composition and key active components of alarm pheromone differentiate among aphid species. Here we conducted a detailed analysis of the terpenoid compounds in the vetch aphid Megoura viciae and its host plant Pisum sativum by using gas chromatography-mass spectrometry. The results showed that a variety of terpenoid compounds existed in the aphid, with four major terpene components, i.e., (-)- $\beta$-pinene (49.74\%), (E)- $\beta$-farnesene (32.64\%), (-)-a-pinene (9.42\%) and (+)-limonene $(5.24 \%)$, in addition to a trace amount of minor terpenoid components $(3.14 \%)$. In contrast, the terpenoid compounds were relatively scarce in the host plant, mainly consisting of squalene (66.13\%) and its analogue 2,3-epoxysqualene (31.59\%) in addition to some minor components. Quantitative analysis of the dynamics of four major terpene components during different developmental stages showed that the monoterpenes increased with continuous development, while the sesquiterpene reached peak at the 3rd instar; all terpene components remained at a high level in the 4th -instar, with (-)- $\beta$-pinene accounting for the highest proportion during all developmental stages. Behavioral assays with single components and mixtures at different concentrations were conducted in a three-compartment olfactometer, revealing that the repellent activities of single components varied in a concentration-dependent manner, but two mixtures (1:44.4:6.5:2.2 and 1:18.4:1.3:0.8) prepared according to the proportions of four major components at the 3rd - and 4th -instar stages maintained a significant repellent activity at all concentrations tested. Our results suggested that (-)-a-pinene and (-)- $\beta$-pinene were the major active components of alarm pheromone in $M$. viciae, but the mixtures of single components play a key role in the alarm behavior of $M$. viciae. Our study helps to understand the chemical ecology of insects and design alternative control strategies against aphids.

\section{Introduction}

Insects use chemical volatiles to communicate in mating, aggregation, predation, alarm and self-defense (Belén et al., 2015). Among them, alarm pheromones as the second largest family of insect pheromones play an important ecological role in insects (Verheggen et al., 2010). Most aphids release alarm pheromones when they are attacked by natural enemies, and both aphid nymphs and adults utilize alarm pheromone to warn con-specifics of danger (Kunert et al., 2007). Aphids are among the most widespread and harmful agricultural pests in the world (Simon and Peccoud, 2018). Previous studies showed that the major component of alarm pheromone for most aphid species is the sesquiterpene ( $E$ )- $\beta$-farnesene (Edwards et al., 1973; Pickett and Griffiths, 1980). Francis et al. (2005) tested the composition of volatile molecules in 23 aphid species, finding that $(E)-\beta$-farnesene was the main component in 16 species and the minor component in five species; only two aphid species (Euceraphis punctipennis and Drepanosiphum platanoides) did not release $(E)-\beta$-farnesene. They also reported a particular profile of volatile molecules composed of not only (E)- $\beta$-farnesene but also several monoterpenes in the vetch aphid Megoura viciae Buckton (Aphididae: Hemiptera), including (-)-a-pinene, (-)- $\beta$-pinene and (+)-limonene, but no behavioral assays were performed. Nevertheless, Bruno et al. (2018) assessed the behavioral response of $M$. viciae to the compounds identified by Francis et al. (2005), indicating that (-)-a-pinene and (+)-limonene were the main active components of alarm pheromone in $M$. viciae. Moreover, they tested a mixture at the ratio of (E)- $\beta$-farnesene $(14.2 \%),(-)$ - $\alpha-$ 
pinene $(11.8 \%)$ and $\beta$-pinene $(74 \%)$ as reported (Table 51$)$, showing a repellent activity against $M$. viciae. Additionally, molecular studies revealed that the recombinant odorant binding protein MvicOBP3 could bind to all four alarm pheromone components of $M$. viciae, displaying a much higher affinity for $(E)$ - $\beta$-farnesene $\left(K_{i} 0.1 \mu \mathrm{M}\right)$ than for $\beta$-pinene $\left(K_{i} 2.3 \mu \mathrm{M}\right),(-)$-a-pinene $\left(K_{i} 1.8 \mu \mathrm{M}\right)$ and (+)-limonene $\left(K_{i} 2.5 \mu M\right)$ (Northey et al., 2016). It seems that the molecular binding affinity could not reflect the alarm activity of terpene components.

M. viciae feeds exclusively on the Fabaceae (Nuessly et al., 2004), causing serious damage to the broad bean Vicia faba and the pea Pisum sativum (Kunert et al., 2008; Leroy et al., 2011). Its unique composition of terpene components differentiate it from most other aphid species in alarm behavior. It has been shown that alarm pheromone was synthesized by the aphid itself in the cotton aphid Aphis gossypii by rearing aphids with artificial diets (Sun and Li, 2017). However, it is still unclear whether other aphids also synthesize de novo alarm pheromone. Our group has been working on the biosynthetic mechanisms of aphid alarm pheromone, yet the major component of alarm pheromone in all aphid species that have been investigated was (E)- $\beta$-farnesene, including the green peach aphid Myzus persicae (Cheng and Li, 2018; Zhang and Li, 2008; Zhang and Li, 2012), A. gossypii (Ma et al., 2010; Sun and Li, 2017; Sun and Li, 2018) and the bird cherry-oat aphid Rhopalosiphum padi (Sun and Li, 2012; Sun and Li, 2019; Sun and Li, 2020). Thus, $M$. viciae can provide a good opportunity for comparative study in this line of work. Here we first analyzed the composition of terpenoid compounds in M. viciae and its host plant Pisum sativum by using gas chromatography-mass spectrometry. Next, the dynamics of the major terpene components was investigated during different developmental stages of $M$. viciae. Moreover, we conducted a series of behavioral assays with single components and mixtures at different concentrations in a three-compartment olfactometer. Our study identified a novel set of alarm pheromones in $M$. viciae.

\section{Materials And Methods}

\section{Culture of aphids}

The aphid M. viciae was provided by the Laboratory of Biological Control led by Dr. Tinghui Liu in Hebei Agricultural University and maintained on P. sativum in the Laboratory of Insect Molecular Ecology in China Agricultural University. The aphids were reared in a climate incubator (RXZ-300B, Ningbo, China) under the conditions of $19 \pm 1{ }^{\circ} \mathrm{C}, 70 \pm 5 \%$ relative humidity and a photoperiod of $16 \mathrm{~L}: 8 \mathrm{D}$.

\section{Collection of terpenoid compounds fromM. viciaeand its host plantP. sativum}

M. viciae aphids (overlapping developmental stages, $n=200$ ) were collected and put into a $1.5-\mathrm{mL}$ centrifuge tube containing $500 \mu \mathrm{L}$ of $n$-hexane on ice, fully milled, centrifuged at $4{ }^{\circ} \mathrm{C}$ for $30 \mathrm{~min}$. The supernatant was transferred into a 2-mL vial for gas chromatography-mass spectrometry (GC-MS) analysis. The same procedure was performed to collect volatile terpenoid compounds from $P$. sativum seedlings (2.0g) for GC-MS analysis. 


\section{Identification of terpenoid compounds fromM. viciaeandP. sativumby GC-MS}

The samples collected from M. viciae and P. sativum were analyzed on an Agilent 6890 gas chromatographer coupled to an Agilent 5973 ion trap mass detector (Agilent Technologies Inc., California, USA). The instrument was equipped with a HP-5 capillary column ( $300 \mathrm{~mm} \times 0.25 \mathrm{~mm} \times 0.25 \mu \mathrm{m}$, Agilent, Santa Clara, USA). The program of GC-MS was set up as described (Huang et al., 2013). Briefly, after sample injection, the GC oven temperature was held at $40^{\circ} \mathrm{C}$ for $1 \mathrm{~min}$, followed by a two-step temperature increase: the first increase was from $40^{\circ} \mathrm{C}$ to $130^{\circ} \mathrm{C}$ at a rate of $4^{\circ} \mathrm{C} / \mathrm{min}$ and maintained for $5 \mathrm{~min}$, and then the temperature was increased at a rate of $10^{\circ} \mathrm{C} / \mathrm{min}$ to $250^{\circ} \mathrm{C}$ and held for $5 \mathrm{~min}$. The temperatures of injector and ion source were $250^{\circ} \mathrm{C}$. The mass spectrometer was operated under the electron impact ionization mode (El, $70 \mathrm{eV}$ ) with a $\mathrm{m} / \mathrm{z}$ scan range of 35-650. Terpenes were identified by comparing their retention time and mass spectra with those of standards (SigmaAldrich, Oakville, Canada) under the same conditions. The quantity of each component was estimated based on the peak area ratio of sample to the chromatographically pure external standard (-)- $\beta$-pinene (Magdalena and Henryk, 2016). Three biological replicates were performed for each treatment. The proportions of single components were calculated as their percentages in total terpenoid compounds.

\section{Quantitative analysis of terpene components at different developmental stages of aphid}

M. viciae aphids of the same developmental stage $(n=30)$, including the 1 st -instar, 2 nd -instar, $3 r d$-instar, 4 th -instar nymphs and adult, were ground in a 1.5-mL centrifuge containing $100 \mu \mathrm{L}$ of hexane. The supernatant was transferred to a chromatography vial for GC/MS analysis as described above. (-)- $\beta$-Pinene (purity >99\%; Sigma-Aldrich, Oakville, Canada) was used as the external standard. Three biological repetitions were performed for each stage. The amount and proportion of each single terpene component were estimated based on the peak area ratio of sample to standard for different developmental stages.

\section{Behavioral assays}

Behavioral assay experiments were carried out in a three-compartment perspex olfactometer modified from the design based on an optimized behavioral selection model of aphid (Khashaveh et al., 2020; Satyajeet et al., 2021; Yu et al., 2019) (Fig. 1). The olfactometer was composed of three compartments $(7 \mathrm{~cm} \times 13 \mathrm{~cm} \times$ $5 \mathrm{~cm}$ for each) connected by a door $(3 \mathrm{~cm} \times 3 \mathrm{~cm})$ between two adjacent compartments. The test samples, i.e., (-)-a-pinene, (-)- $\beta$-pinene, $(+)$-limonene and $(E)-\beta$-farnesene, were diluted into three different concentrations $(0.1 \mu \mathrm{g} / \mu \mathrm{L}, 1 \mu \mathrm{g} / \mu \mathrm{L}$ and $10 \mu \mathrm{g} / \mu \mathrm{L}$ ) with light mineral oil. Meanwhile, two mixtures (Mix I and Mix II) were prepared by mixing the test samples in that order in the ratios of 1:44.4:6.5:2.2 and

1:18.4:1.3:0.8 according to the proportions of the four major terpene components at the 3rd - and 4th -instar nymphal stages, respectively. Light mineral oil alone was used as a negative control. The test sample (B) and control $(C)$ were placed in a petri dish $(\$ 3.0 \mathrm{~cm})$ near the door of the side compartments, respectively, and five host plants fixed in smaller petri dishes $(\Phi 1.0 \mathrm{~cm}$ ) (covered with $10 \%$ agar) were placed in the far side of the lateral compartments. Twenty wingless 3rd - and 4th -instar nymphs were introduced into the petri dish in the middle (A) and allowed to move freely for $30 \mathrm{~min}$. A total of 100 nymphs were used to test 
their selection preference for each sample compound. All behavioral assays were performed under the same conditions and under a dark environment to avoid light interference. The numbers of aphids crawling close to the host plants in the two lateral compartments were counted. For each sample tested, the behavioral index value (BIV) was calculated according to the following formula: BIV $=[(C-T) /(C+T)] \times 100$, where $C$ and $T$ are the numbers of aphids in the control and treatment compartments, respectively.

\section{Data analysis}

The quantities of (-)-a-pinene, (-)- $\beta$-pinene and (+)-limonene and $(E)$ - $\beta$-farnesene were statistically analyzed and compared on the GraphPad Statistics version 8.0 (San Diego, USA) using One-way analysis of variance (ANOVA) followed by Tukey's B multiple range test $(P<0.05)$. The significance of differences in the behavioral index values were analyzed on SPSS Statistics version 21 (IBM) using ANOVA followed by Duncan's test $(P<0.05)$.

\section{Results}

\section{Identification of the terpenoid compounds from M. viciae and its host plant $P$. sativum}

The terpenoid compounds from $M$. viciae and its host plant $P$. sativum were identified by using GC-MS analysis. The results showed that four major terpene compounds were detected in $M$. viciae, including the sesquiterpene (E)- $\beta$-farnesene and three monoterpenes, i.e., (-)-a-pinene, (-)- $\beta$-pinene and (+)-limonene (Table 1). The compounds (-)-a-pinene, (-)- $\beta$-pinene, (+)-limonene and $(E)-\beta$-farnesene were corresponding to the peaks nos.1, 2, 3 and 6, respectively, as characterized by GC-MS (Fig. 2 and Fig. S1). Interestingly, some additional minor peaks were detected, which were identified as $\beta$-myrcene, $(+)$-sabinene, camphene and aterpineol, based on comparative analysis with the standard mass spectrometry library NIST17s. Moreover, the proportions of different components were calculated: (-)-a-pinene (9.42\%), (-)- $\beta$-pinene $(49.74 \%)$ and (+)limonene (5.24\%), and (E)- $\beta$-farnesene (32.64\%), with the minor components accounting for $3.14 \%$ (Fig. 3A). In contrast, the types of terpenoid compounds were relatively scarce in the host plant compared to in the aphid, mainly consisting of squalene (66.13\%) and its analogue 2,3-epoxysqualene (31.59\%) (Table 2, Fig. 3B and Fig. S2). Some minor terpenoid components were also identified in $P$. sativum, such as 4isopropyl-5-methylhexa-2,4-dien-1-ol, 2,4-pentadien-1-ol, 3-pentyl-, (2Z)-, limonene and cyclohexanol, 1methyl-4-(1-methylethenyl)-, cis-, based on comparative analysis with the standard library. 
Table 1

GC-MS identification of terpenoid compounds in Megoura viciae

\begin{tabular}{|llllllllll|}
\hline No. & $\begin{array}{l}\text { Retention } \\
\text { time (min) }\end{array}$ & $\mathbf{m} / \mathbf{z}$ & $\begin{array}{l}\text { Peak } \\
\text { area }\end{array}$ & $\begin{array}{l}\% \\
\text { Peak } \\
\text { area }\end{array}$ & $\begin{array}{l}\text { Peak } \\
\text { height }\end{array}$ & $\begin{array}{l}\% \\
\text { Peak } \\
\text { height }\end{array}$ & $\begin{array}{l}\text { Name of } \\
\text { terpenoid } \\
\text { compound }\end{array}$ & $\begin{array}{l}\text { Molecular } \\
\text { formula }\end{array}$ \\
\hline 1 & 10.099 & TIC & 1203409 & 0.18 & 456923 & 0.24 & $(-)$-a-Pinene & C10H16 \\
\hline 2 & 10.639 & TIC & 57434 & 0.01 & 14470 & 0.01 & Camphene & C10H16 \\
\hline 3 & 11.562 & TIC & 75660 & 0.01 & 30386 & 0.02 & $(+)$-Sabinene & C10H16 \\
\hline 4 & 11.676 & TIC & 6325841 & 0.95 & 2149907 & 1.15 & $(-)-\beta$-Pinene & C10H16 \\
\hline 5 & 12.244 & TIC & 229816 & 0.03 & 68879 & 0.04 & $\beta-$-Myrcene & C10H16 \\
\hline 6 & 13.665 & TIC & 658790 & 0.1 & 195955 & 0.1 & $(+)$-Limonene & C10H16 \\
\hline 7 & 19.977 & TIC & 40572 & 0.01 & 8983 & 0 & a-Terpineol & C10H180 \\
\hline 8 & 29.105 & TIC & 4102715 & 0.62 & 805659 & 0.43 & $(E)-\beta$-Farnesene & C15H24 \\
\hline
\end{tabular}

Table 2

GC-MS identification of terpenoid compounds in the host plant Pisum sativum

\begin{tabular}{|lllllllll|}
\hline No. & $\begin{array}{l}\text { Retention } \\
\text { time } \\
\text { (min) }\end{array}$ & $\mathbf{m} / \boldsymbol{z}$ & Peak area & $\begin{array}{l}\% \\
\text { Peak } \\
\text { area }\end{array}$ & $\begin{array}{l}\text { Peak } \\
\text { height }\end{array}$ & $\begin{array}{l}\text { \% } \\
\text { Peak } \\
\text { height }\end{array}$ & Name of terpenoid & $\begin{array}{c}\text { Molecular } \\
\text { formula }\end{array}$ \\
\hline 1 & 13.674 & TIC & 138863 & 0.02 & 30615 & 0.02 & Limonene & C10H16 \\
\hline 2 & 22.52 & TIC & 42633 & 0.01 & 15660 & 0.01 & $\begin{array}{l}\text { Cyclohexanol, 1- } \\
\text { methyl-4-(1- }\end{array}$ & C10H180 \\
methylethenyl)-, cis- & \\
\hline 3 & 27.43 & TIC & 174315 & 0.03 & 83769 & 0.05 & $\begin{array}{l}\text { 2,4-Pentadien-1- } \\
\text { ol, 3-pentyl-, (2Z)- }\end{array}$ & C10H180 \\
\hline 4 & 42.391 & TIC & 1057080 & 0.18 & 267448 & 0.15 & $\begin{array}{l}\text { 4-Isopropyl-5- } \\
\text { methylhexa-2,4- }\end{array}$ & C10H180 \\
\hline 5 & 42.944 & TIC & 19187774 & 3.32 & 3786188 & 2.14 & 2,3-Squalene-epoxy & C30H500 \\
\hline 6 & 43.013 & TIC & 13851345 & 2.39 & 3818540 & 2.16 & Squalene & C30H50 \\
\hline 7 & 43.094 & TIC & 26363816 & 4.56 & 5569989 & 3.15 & Squalene & C30H50 \\
\hline
\end{tabular}

\section{Quantitative dynamics of terpene compounds at different developmental stages of M. viciae}

The contents of four major terpene components were investigated at different developmental stages of aphid: 1st -instar, 2nd -instar, 3rd -instar, 4th -instar and adult. The results showed that the content of (-)- $\beta$ - 
pinene increased rapidly from the 1 st - to 2 nd -instars, and the content of $(E)$ - $\beta$-farnesene had a distinct increase from the 2 nd - to 3 rd -instars, while the contents of (+)-limonene and (-)-a-pinene had a substantial increase from the 3rd - to 4th -instars (Fig. 4 top). All components remained at a high level in the 4th -instar nymph. As a general trend, the contents of all monoterpene components increased with continuous development, while the content of $(E)$ - $\beta$-farnesene displayed a different trend. The proportions of different terpenoid components were also calculated for different developmental stages (Fig. 4 bottom), showing that the proportion of (-)- $\beta$-pinene was the highest $(>81 \%)$ from the 2 nd -instar to adult stages. The ratios of the four major terpene components at the $3 \mathrm{rd}$ - and 4 th -instar stages were used for preparing the two mixtures for behavioral assays.

\section{Behavioral responses of $M$. viciae to single and mixed terpene compounds}

The behavioral responses of $M$. viciae to single terpene components and their mixture were measured in the three-compartment olfactometer. The two mixtures of (-)-a-pinene, (-)- $\beta$-pinene, $(+)$-limonene and $(E)-\beta$ farnesene were prepared in that order at the ratios of 1:44.4:6.5:2.2 (Mix I) and 1:18.4:1.3:0.8 (Mix II) according to the proportions of the four major terpene components at the 3rd - and 4th -instar stages. Behavioral responses were categorized as four types: NR (no response, BIV $<20 \%$ ), W (weak, $20<$ BIV $<40 \%$ ), M (moderate, $40 \%<$ BIV < 60\%) and S (strong, BIV > 60\%) (Hieu et al., 2014; Khashaveh et al., 2020). The results showed that the repellent activities of the compounds tested varied at different concentrations (Table 3 and Fig. 5). Specifically, (-)-a-pinene, (-)- $\beta$-pinene, (+)-limonene and ( $E$ )- $\beta$-farnesene all displayed repellent activity to some extents at the concentration of $10.0 \mu \mathrm{g} / \mu \mathrm{L}$ : (-)-a-pinene had the strongest activity with a highest BIV value (Type S); (-)- $\beta$-pinene, Mix I and Mix II exhibited moderate repellent activity (M), and $(+)$-limonene and (E)- $\beta$-farnesene showed merely weak activity $(W)$. Similarly, under the concentration of 1.0 $\mu \mathrm{g} / \mu \mathrm{L},(-)$-a-pinene, (-)- $\beta$-pinene, Mix I and Mix II all showed moderate repellent activity, and (+)-limonene and $(E)-\beta$-farnesene had only weak repellent activity. In contrast, under the concentration of $0.1 \mu \mathrm{g} / \mu \mathrm{L}$, no behavioral response or merely weak repellent activity were observed for all four single compounds. As a single component, $(E)$ - $\beta$-farnesene displayed only weak repellent activity at all tested concentrations. Nevertheless, the two mixtures showed a significant repellent activity against $M$. viciae $\left(F_{17,72}=5.748, P<\right.$ $0.05)$ at the low concentration of $0.1 \mu \mathrm{g} / \mu \mathrm{L}$. 
Table 3

Behavioral index values (BIV) of Megoura viciae in response to single and mixed terpene compounds

\begin{tabular}{|c|c|c|c|}
\hline Compound & Concentration $(\mu \mathrm{g} / \mu \mathrm{L})$ & BIV (\%) & BA \\
\hline (-)-a-Pinene & \multirow[t]{6}{*}{10.0} & $67.85 \pm 4.52 \mathrm{a}$ & Repellent (S) \\
\hline (-)-ß-Pinene & & $42.55 \pm 9.52$ abcde & Repellent (M) \\
\hline (+)-Limonene & & $23.49 \pm 6.37 \mathrm{cde}$ & Repellent (W) \\
\hline (E)- $\beta$-farnesene & & $20.33 \pm 2.43$ cde & Repellent (W) \\
\hline Mix I & & $58.19 \pm 2.69 \mathrm{ab}$ & Repellent (M) \\
\hline Mix II & & $43.55 \pm 8.39$ abcde & Repellent (M) \\
\hline (-)-a-Pinene & \multirow[t]{6}{*}{1.0} & $34.81 \pm 12.51$ bcde & Repellent (M) \\
\hline (-)- $\beta$-Pinene & & $38.67 \pm 4.94$ abcde & Repellent (M) \\
\hline (+)-Limonene & & $23.49 \pm 6.39 \mathrm{cde}$ & Repellent (W) \\
\hline$(E)-\beta$-farnesene & & $27.94 \pm 6.28$ bcde & Repellent (W) \\
\hline Mix I & & $43.59 \pm 4.80$ abcde & Repellent (M) \\
\hline Mix II & & $49.00 \pm 7.14 \mathrm{abcd}$ & Repellent (M) \\
\hline (-)-a-Pinene & \multirow[t]{6}{*}{0.1} & $16.41 \pm 8.73 \mathrm{de}$ & NR \\
\hline (-)-ß-Pinene & & $12.89 \pm 6.34 \mathrm{e}$ & NR \\
\hline (+)-Limonene & & $13.27 \pm 2.45 \mathrm{e}$ & $N R$ \\
\hline$(E)-\beta$-farnesene & & $22.53 \pm 1.56 \mathrm{~cd}$ & Repellent (W) \\
\hline Mix I & & $51.27 \pm 3.94 \mathrm{abc}$ & Repellent (M) \\
\hline Mix II & & $40.95 \pm 1.90$ abcde & Repellent (M) \\
\hline \multicolumn{4}{|c|}{ 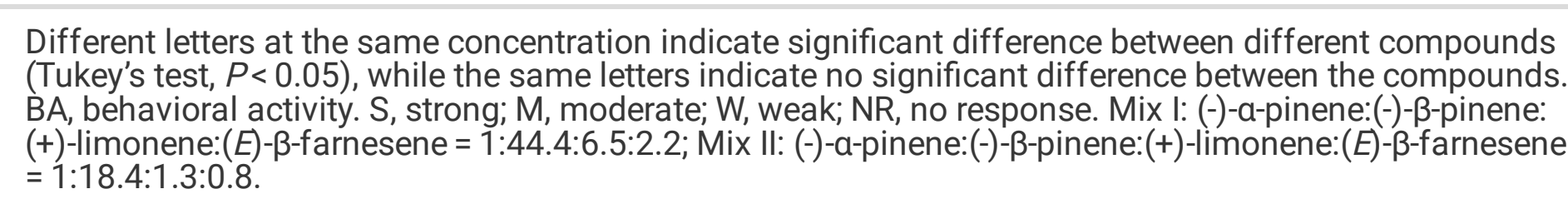 } \\
\hline
\end{tabular}

\section{Discussion}

GC-MS analysis of the terpenoid compounds in M. viciae identified four major terpene components, including (-)- $\beta$-pinene (49.74\%), (E)- $\beta$-farnesene (32.64\%), (-)-a-pinene (9.42\%) and (+)-limonene (5.24\%), in addition to some minor terpenoid components (3.14\%). Compared with the previously reported data (Francis et al., 2005), the proportion of ( $E$ )- $\beta$-farnesene measured here was much higher ( $32.64 \%$ vs $14.2 \%$ ), while the proportion of (-)- $\beta$-pinene was much lower (49.74\% vs $74.0 \%)$. The aphid samples used for GC-MS analysis 
were composed of winged and wingless forms at different developmental stages in both studies. Thus the difference in the proportions of terpenoid components in $M$. viciae might be caused by either endogenous or exogenous factors. As a possible exogenous factor, the devices used in two studies were different; as the endogenous factor, the aphids analyzed were different: it was highly probable that the compositions of terpenoid components differentiated in the two different geographic populations of $M$. viciae. Therefore, the ecological significance of our results needs further investigation.

Quantitative analysis of the dynamics of the four major terpene components in M. viciae during different developmental stages revealed different patterns of change trend in the monoterpene and sesquiterpene components: the monoterpenes increased with continuous development, while the latter reached peak at the $3 \mathrm{rd}$-instar. We also found that all terpenoid components remained at a high level in the 4 th -instar; the proportion of (-)- $\beta$-pinene remained the highest (> 81\%) from the 2 nd -instar to adult stages. This is the first report of the temporal dynamics in the composition of terpenoid components in an aphid species. These data formed the basis for the preparation of volatile mixtures for olfactory choice assays.

Behavioral assays revealed that the repellent activities of single components were concentration-dependent but the mixtures not: all single components showed a repellent activity against $M$. viciae to some extents at $1.0 \mu \mathrm{g} / \mu \mathrm{L}$ or above, but displayed no or merely weak activity at $0.1 \mu \mathrm{g} / \mu \mathrm{L}$. (-)-a-pinene showed a strong repellent activity at $10.0 \mu \mathrm{g} / \mu \mathrm{L}$, a moderate activity at $1.0 \mu \mathrm{g} / \mu \mathrm{L}$, but no response at $0.1 \mu \mathrm{g} / \mu \mathrm{L}$, while the two mixtures showed a moderate repellent activity against $M$. viciae at all tested concentrations. Under all concentrations tested, $(+)$-limonene and $(E)$ - $\beta$-farnesene displayed only weak activity or no response. Our results suggested that (-)-a-pinene and (-)- $\beta$-pinene were the major active components of alarm pheromone in $M$. viciae, but the volatile mixtures of the single terpene components in a specific ratio play a key role in the alarm behavior of $M$. viciae, as a moderate repellent activity remained in the mixtures at $0.1 \mu \mathrm{g} / \mu \mathrm{L}$ although not in single components. In a previous study, five terpene compounds, i.e., (-)-a-pinene, $( \pm)-\mathrm{a}-$ pinene, $\beta$-pinene, $(+)$-limonene and $(E)-\beta$-farnesene, were tested, showing that $( \pm)$ - $\alpha$-pinene, $\beta$-pinene and $(E)$ $\beta$-farnesene as single components were not repellent against $M$. viciae, although (-)-a-pinene, (+)-limonene and a mixture containing $14.2 \%(E)-\beta$-farnesene, $11.8 \%$ (-)-a-pinene, $74 \% \beta$-pinene showed a significant repellent activity against the aphids (Bruno et al., 2018). Their data indicated that (-)-a-pinene and (+)limonene were the major active components of alarm pheromone in $M$. viciae, and similarly, the mixture in a ratio prepared according to the proportions of different terpenoid compounds determined by Francis et al. (2005) played a significant role in the alarm behavior of M. viciae. The finding that (-)-a-pinene was among the major alarm pheromone components was consistent between our study and the previous study; what's new in our study is that we found a concentration-dependent manner of the alarm pheromone components and the synergistic action mode of single components based on the formulae calculated according to the proportions of single components at the 3rd - and 4th -instar stages when the aphids might be most responsive to alarm pheromone. Additionally, the previous study used a Y-tube olfactometer, while we used a three-compartment olfactometer. Our device simulated the natural selection environment by adding the host plants in the lateral compartments (Yu et al., 2019).

Last but not least, our results revealed that the terpenoid compounds were relatively scarce in the host plant $P$. sativum, containing none of the major aphid alarm pheromone components. This result added a 
biochemical evidence to the notion that alarm pheromone was synthesized de novo in the aphid (Sun and Li, 2017), and it is unlikely that aphid alarm pheromone is taken directly from the host plant.

In summary, we identified four major terpene components in addition to some minor terpenoid components in M. viciae. Different types of components exhibited different patterns of change trend across the developmental process of aphid: the monoterpenes increased with continuous development, while the sesquiterpene reached peak at the 3rd -instar; all terpene components remained at a high level in the 4th instar, with (-)- $\beta$-pinene accounting for the highest proportion during all developmental stages. Behavioral assays revealed that the repellent activities of single components varied in a concentration-dependent manner, but the mixtures maintained a significant repellent activity at all concentrations tested. Our results suggested that (-)-a-pinene and (-)- $\beta$-pinene were the major active components of alarm pheromone in $M$. viciae, but the mixtures of single components play a key role in the alarm behavior of $M$. viciae. Our study helps to understand the chemical ecology of insects and design alternative control strategies against aphids.

\section{Declarations}

\section{Acknowledgements}

We kindly acknowledge Dr. Tinghui Liu (Hebei Agricultural University) for providing the aphid stock of $M$. viciae. This work is supported by the National Natural Science Foundation of China (Grant nos. 31972267 and 31772169).

\section{Funding}

Dr. Li ZX received funding from the National Natural Science Foundation of China (Grant nos. 31972267 and 31772169).

\section{Data Availability}

The data sets generated during and/or analyzed during the present study are available from the corresponding author. Raw data are partially included in the Suppl. Info. file.

\section{Code Availability}

Not applicable.

\section{Ethics Approval}


The study used insects, but none of the experiments raise ethical issues. All animal care and experimentation complied with the guidelines provided by the Association for the Study of Animal Behavior (ASAB) and the Animal Behavior Society (ABS).

\section{Consent to Participate}

Not applicable.

\section{Consent for Publication}

Not applicable.

\section{Conflicts of Interest/Competing Interests}

The authors have no conflicts of interest or competing interests to disclose.

\section{References}

1. Belén C, Linda Marie R, Maria B, Hans RN, Nicolai V, Meyling BR, Peter A (2015) Habitat selection of a parasitoid mediated by volatiles informing on host and intraguild predator densities. Oecologia 179:151-162. https://doi.org/10.1007/ s00442-015-3326-2

2. Bruno D, Grossi G, Salvia R, Scala A, Farina D, Grimaldi A, Zhou JJ, Bufo SA, Vogel H, Grosse-Wilde E, Hansson BS, Falabella P (2018) Sensilla morphology and complex expression pattern of odorant binding proteins in the vetch aphid Megoura viciae (Hemiptera: Aphididae). Front Physiol 9:777. https://doi.org/10.3389/fphys.2018.00777

3. Cheng YJ, Li ZX (2018) Spatiotemporal expression profiling of the farnesyl diphosphate synthase genes in aphids and analysis of their associations with the biosynthesis of alarm pheromone. Bull Entomol Res 109:398-407. https://doi.org/ 10.1017/S0007485318000706

4. Edwards LJ, Siddall JB, Dunham LL, Uden P, Kislow CJ (1973) Trans- $\beta$-farnesene, alarm pheromone of the green peach aphid, Myzus persicae (sulzer). Nature 241:126-127. https://doi.org/10.1038/241126b0

5. Francis F, Vandermoten S, Verheggen F, Lognay G, Haubruge $E(2005)$ Is the $(E)$ - $\beta$-farnesene only volatile terpenoid in aphids? J Appl Entomol 129:6-11. https://doi.org/10.1111/j.1439-0418.2005.00925.x

6. Hieu TT, Jung JW, Kim SI, Ahn YJ, Kwon HW (2014) Behavioural and electroantennogram responses of the stable fly (Stomoxys calcitrans) to plant essential oils and their mixtures with attractants. Pest Manag Sci 70:163-172. https://doi.org/10.1002/ps.3547

7. Huang XZ, Xiao YT, Köllner TG, Zhang WN, Wu JX, Wu J, Guo, YY, Zhang YJ (2013) Identification and characterization of $(E)$ - $\beta$-caryophyllene synthase and $\alpha / \beta$-pinene synthase potentially involved in 
constitutive and herbivore-induced terpene formation in cotton. Plant Physiol Biochem 73:302-308. https://doi.org/10.1016/ j.plaphy.2013.10.017

8. Khashaveh A, An XK, Shan S, Xiao Y, Wang Q, Wang SN, Li ZB, Geng T, Gu SH, Zhang YJ (2020) Deorphanization of an odorant receptor revealed new bioactive components for green mirid bug Apolygus lucorum (Hemiptera: Miridae). Pest Manag Sci 76:1626-1638. https://doi.org/10.1002/ps.5682

9. Kunert G, Schmoock-Ortlepp K, Reissmann U, Creutzburg S, Weisser WW (2008) The influence of natural enemies on wing induction in Aphis fabae and Megoura viciae (Hemiptera: Aphididae). Bull Entomol Res 98:57-62. https://doi.org/ 10.1017/S0007485307005391

10. Leroy PD, Wathelet B, Sabri A, Francis F, Verheggen FJ, Capella Q, Thonart P, Haubruge E (2011) Aphidhost plant interactions: does aphid honeydew exactly reflect the host plant amino acid composition? Arthropod-Plant Inte 5:193-199. https://doi.org/10.1007/s11829-011-9128-5

11. Ma GY, Sun XF, Zhang YL, Shen ZY (2010) Molecular cloning and characterization of a prenyltransferase from the cotton aphid, Aphis gossypii. Insect Biochem Mol Biol 40:0-561. https://doi.org/10.1016/j.ibmb.2010.05.003

12. Magdalena K, Henryk HJ (2017) In-tube extraction for the determination of the main volatile compounds in Physalis peruviana J Sep Sci 40:532-541. https://doi.org/10.1002/jssc.201600797

13. Northey T, Venthur H, Biasio FD, Chauviac FX, Cole A, Ribeiro KAL, Grossi G, Falabella P, Field LM, Keep LH, Zhou JJ (2016) Crystal structures and binding dynamics of odorant-binding protein 3 from two aphid species Megoura viciae and Nasonovia ribisnigri. Sci Rep 6:24739. https://doi.org/10.1038/srep24739

14. Pickett JA, Griffiths DC (1980) Composition of aphid alarm pheromones. J Chem Ecol 6:349-360. https://doi.org/10.1007/BF01402913

15. Satyajeet G, Anusha LKK, Kaveri D, Jean-Marie B, Renee MB (2021) The scent of life: Phoretic nematodes use wasp volatiles and carbon dioxide to choose functional vehicles for dispersal. J Chem Ecol 47:139-152. https://doi.org/ 10.1007/s10886-21-01242-5

16. Simon JC, Peccoud J (2018) Rapid evolution of aphid pests in agricultural environments. Curr Opin Insect Sci 26:17-24. https://doi.org/10.1016/ j.cois.2017.12.009

17. Sun ZJ, Li ZX (2017) Host plants and obligate endosymbionts are not the sources for biosynthesis of the aphid alarm pheromone. Sci Rep 7:6041. https://doi.org /10.1038/s41598-017-06465-9

18. Sun ZJ, Li ZX (2018) The terpenoid backbone biosynthesis pathway directly affects the biosynthesis of alarm pheromone in the aphid. Insect Mol Biol 27:824-834. https://doi.org/10.1111/imb.12521

19. Sun XF, Li ZX (2012) In silico and in vitro analyses identified three amino acid residues critical to the catalysis of two aphid farnesyl diphosphate synthase. Protein J 31:417-424. https://doi.org/10.1007/s10930-012-9421-x

20. Sun CX, Li ZX (2019) Production of alarm pheromone starts at embryo stage and is modulated by rearing conditions and farnesyl diphosphate synthase genes in the bird cherry-oat aphid Rhopalosiphum padi. Bull Entomol Res 109:821-830. https://doi.org/10.1017/S0007485319000154 
21. Sun CX, Li ZX (2020) Biosynthesis of aphid alarm pheromone is modulated in response to starvation stress under regulation by the insulin, glycolysis and isoprenoid pathways. $\mathrm{J}$ Insect Physiol 128:104174. https://doi.org/10.1016/ j.jinsphys.2020.104174

22. Verheggen FJ, Haubruge E, Mescher MC (2010) Alarm pheromones-chemical signaling in response to danger. Vitam Horm 83:215-239. https://doi.org/10.1016/ S0083-6729(10)83009-2

23. Yu XD, Jia DY, Duan PF (2019) Plasmid engineering of aphid alarm pheromone in tobacco seedlings affects the preference of aphids. Plant Signal Behav 14:1-4.

https://doi.org/10.1080/15592324.2019.1588669

24. Zhang YL, Li ZX (2008) Two different farnesyl diphosphate synthase genes exist in the genome of the green peach aphid, Myzus persicae. Genome 51:501-510. https://doi.org/10.1139/G08-037

25. Zhang YL, Li ZX (2012) Functional analysis and molecular docking identify two active short-chain prenyltransferases in the green peach aphid, Myzus persicae. Arch Insect Biochem Physiol 81:63-76. https://doi.org/10.1002/arch.21032

\section{Figures}

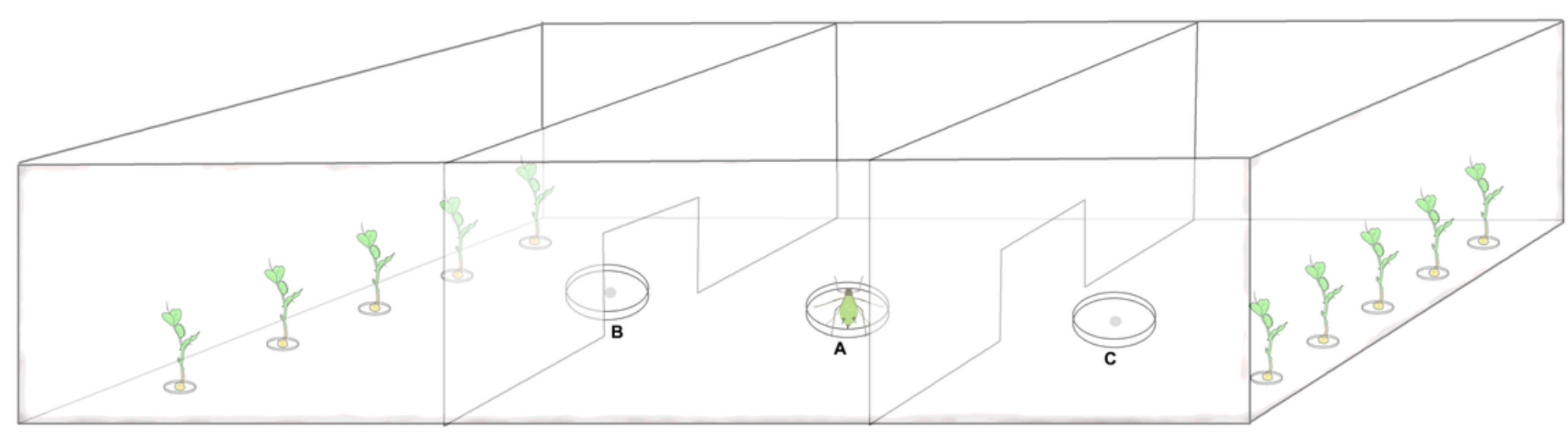

\section{Figure 1}

Composition of terpenoid compounds from M. viciae (A) and its host plant $\mathrm{P}$. sativum (B). The terpenoid components are identified by GC-MS analysis. The proportions of different components are calculated based on the percentages of peak areas. 

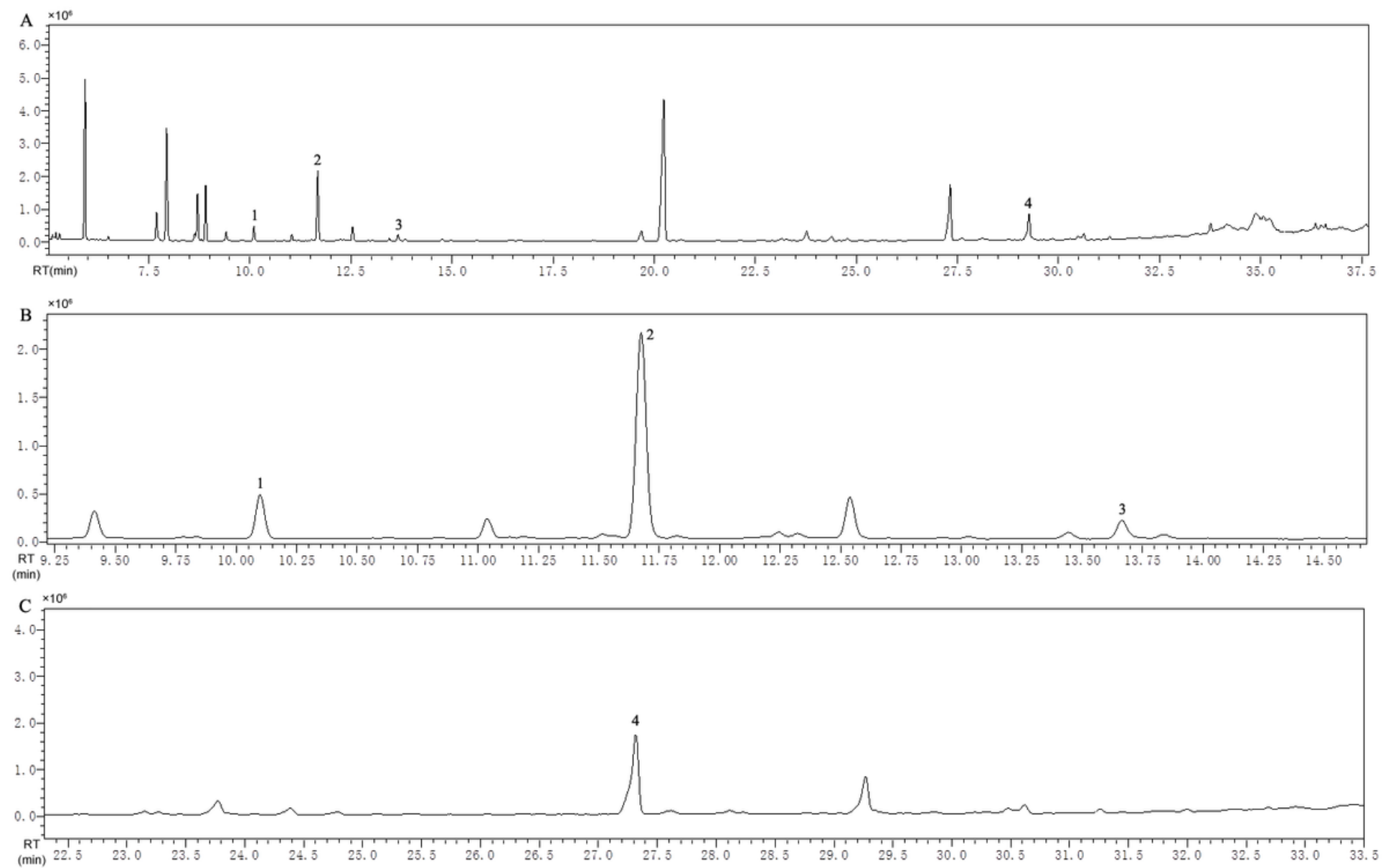

Figure 2

Gas chromatograms of four major terpene compounds from M. viciae. (A) The gas chromatogram of whole component analysis. (B) The local gas chromatogram of monoterpene analysis. (C) Local amplification of the gas chromatogram of (E)- $\beta$-farnesene. The peaks nos. 1, 2, 3 and 4 indicate (-)-a-pinene, (-)- $\beta$-pinene, (+)limonene and (E)- $\beta$-farnesene, respectively.
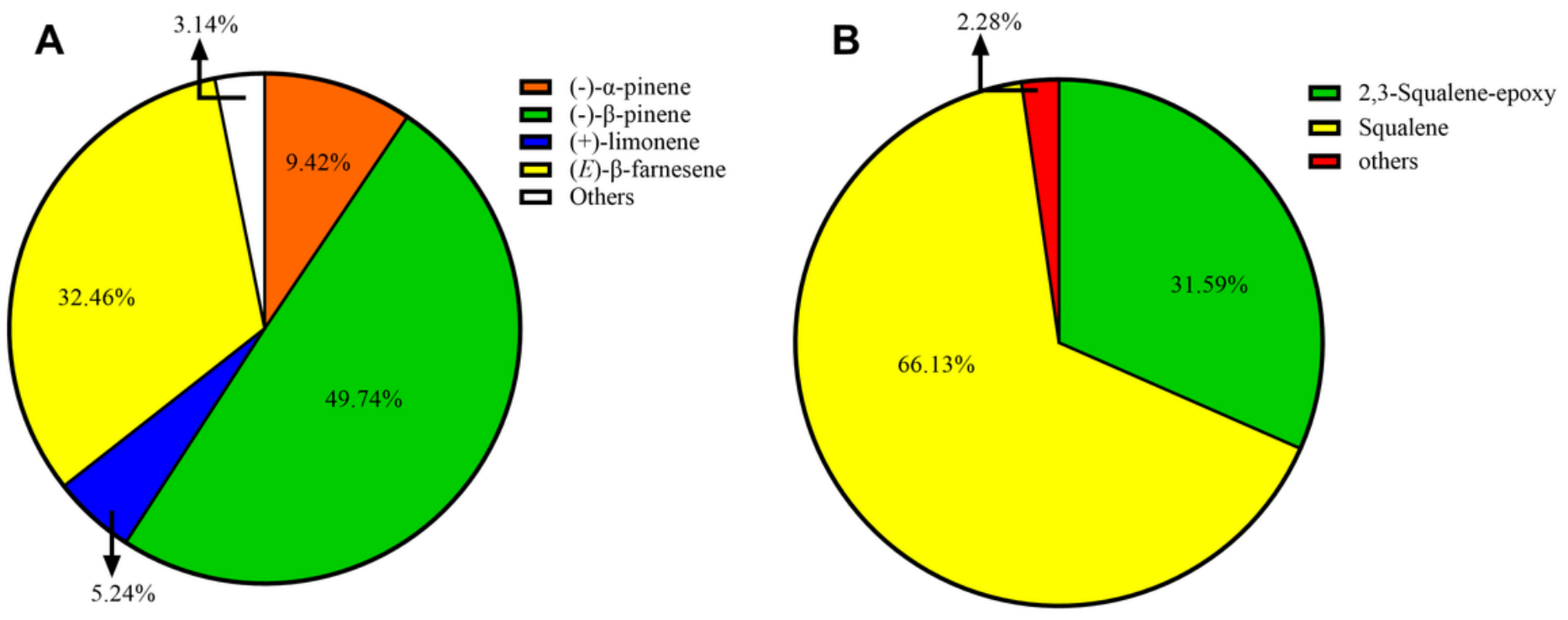
Figure 3

Temporal dynamics of terpene compounds at different developmental stages of M. viciae (top). The proportions of four major terpene components at the 1st-instar, 2nd-instar, 3rd-instar, 4th-instar nymphal and adult stages are also shown (bottom). The proportions of different components are calculated based on the percentages of peak areas.

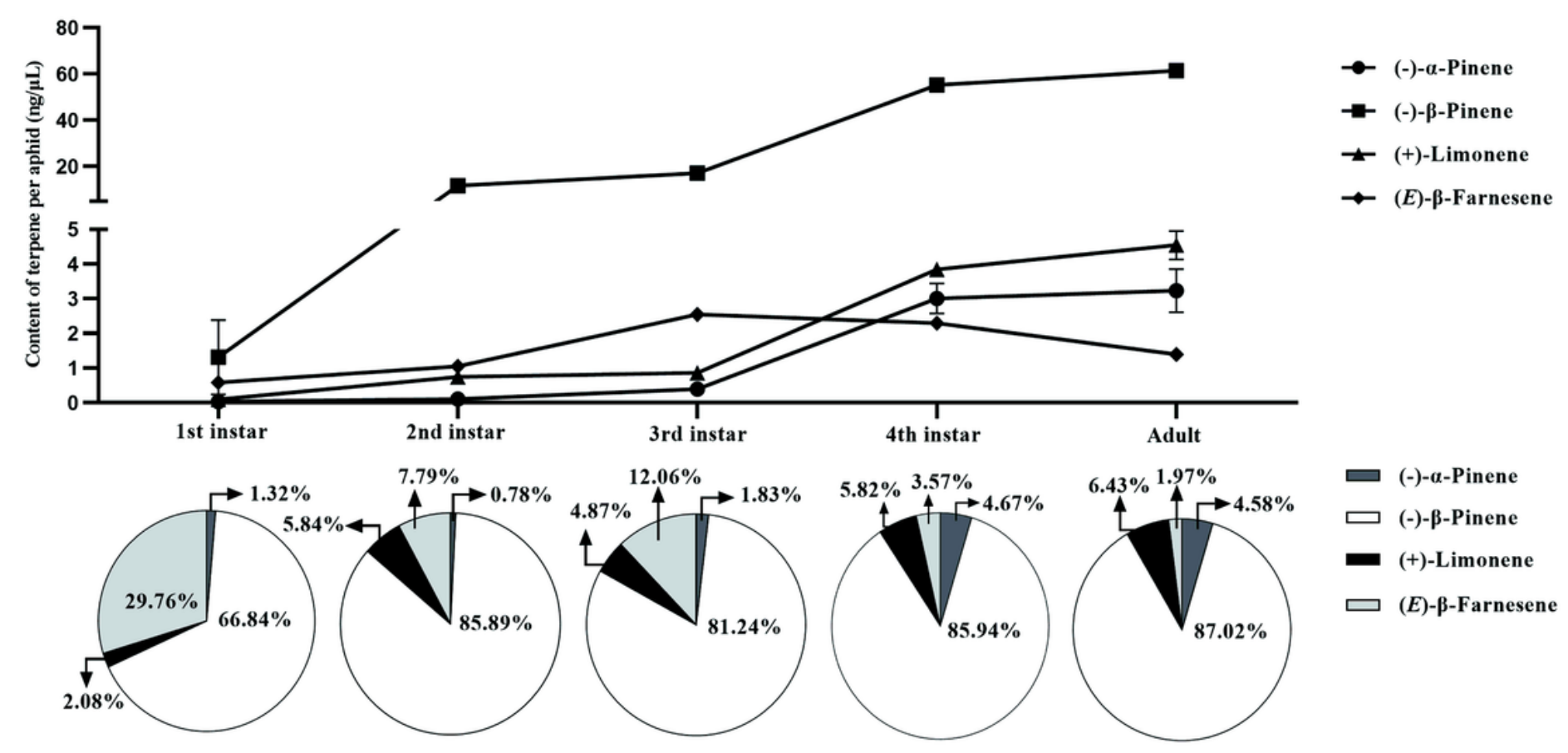

Figure 4

A schematic diagram showing the three-compartment olfactometer for behavioral assays of $M$. viciae. The olfactometer is composed of three compartments $(7 \mathrm{~cm} \times 13 \mathrm{~cm} \times 5 \mathrm{~cm}$ for each). A group of 20 wingless 3rd-4th nymphs are released in Dish A (middle). Filter papers dipped with test compounds and mineral oil (negative control) are placed in Dish B and C, respectively. The numbers of aphids moving near to the host plants in the side compartments are recorded. Five biological repetitions are performed. 


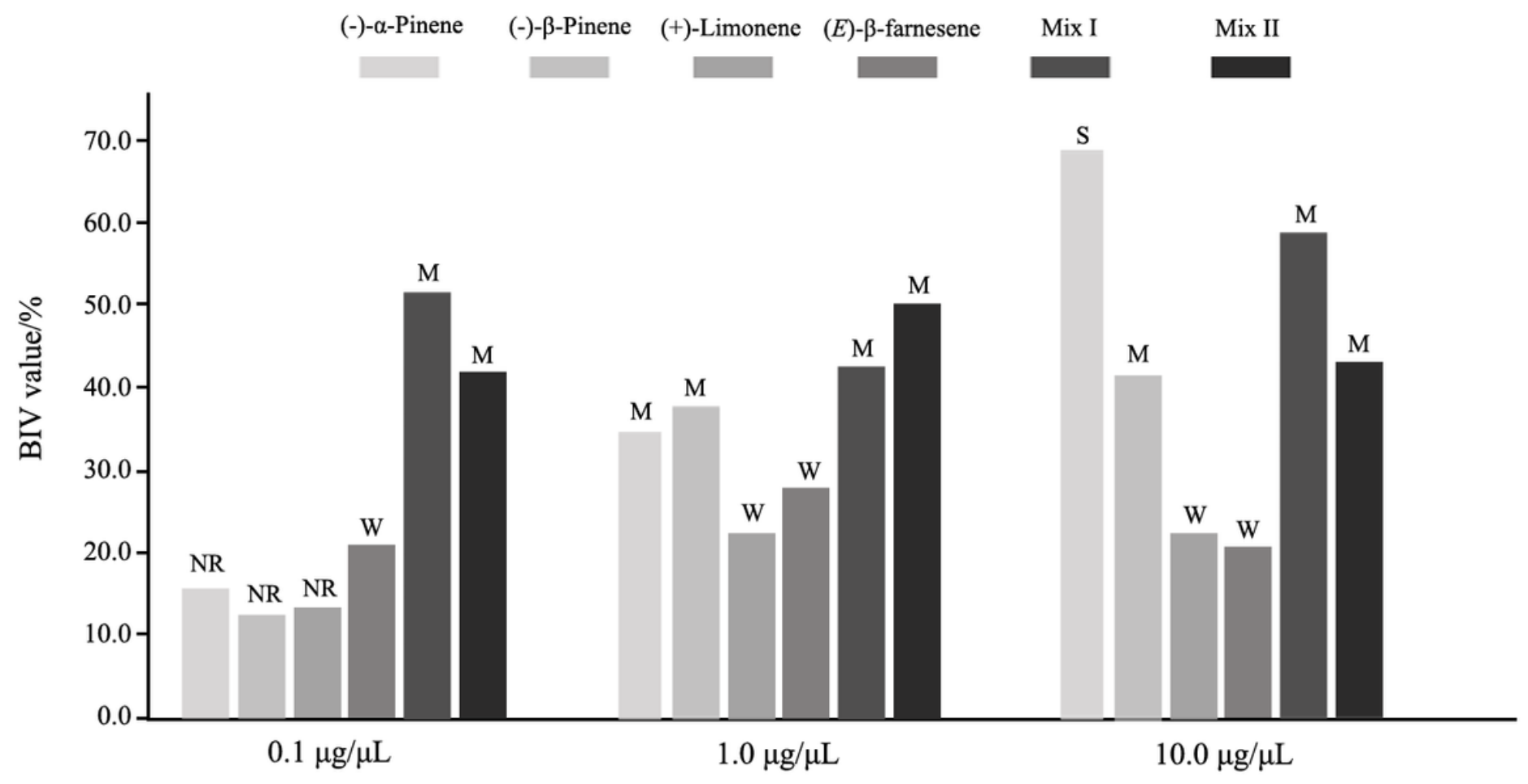

\section{Figure 5}

Behavioral responses of Megoura viciae to single terpene compounds and their mixtures. BIV, behavioral index value. S, strong; $M$, moderate; $W$, weak; NR, no response. Mix I: (-)-a-pinene:(-)- $\beta$-pinene:(+)-limonene: (E)- $\beta$-farnesene=1:44.4:6.5: 2.2; Mix II: (-)-a-pinene:(-)- $\beta$-pinene:(+)-limonene:(E)- $\beta$-farnesene=1:18.4:1.3:0.8.

\section{Supplementary Files}

This is a list of supplementary files associated with this preprint. Click to download.

- Graphicalabstract.tif

- Suppl.Info.docx 